\title{
Sociotechnical Implementation of Prescriptive Maintenance for Onshore Wind Turbines
}

\author{
Bernhard Strack \\ Institute for Industrial Management \\ (FIR) at RWTH Aachen University \\ bernhard.strack@fir.rwth-aachen.de \\ Martin Lenart \\ Institute for Industrial Management \\ (FIR) at RWTH Aachen University \\ martin.lenart@fir.rwth-aachen.de
}

\author{
Jana Frank \\ Institute for Industrial Management \\ (FIR) at RWTH Aachen University \\ jana.frank@ fir.rwth-aachen.de \\ Florian Pfau \\ Institute for Industrial Management \\ (FIR) at RWTH Aachen University \\ florian.pfau@fir.rwth-aachen.de
}

\author{
Volker Stich \\ Institute for Industrial Management \\ (FIR) at RWTH Aachen University \\ volker.stich@fir.rwth-aachen.de
}

\begin{abstract}
Electricity generated by wind turbines (WT) is a mainstay of the transition to renewable energy. In order to economically utilize WT is, operating and maintenance costs, which account for $25 \%$ of total electricity generation costs in onshore WT's, are a focus of cost reduction activities. Implementing a data-driven prescriptive maintenance approach is one way to achieve this. So far, various approaches for prescriptive maintenance for onshore WT's have been suggested. However, little research has addressed the practical implementation considering sociotechnical aspects. The aim of this paper is therefore to identify success factors for the successful implementation of such a maintenance strategy with clear and holistic guidance on how existing knowledge on prescriptive maintenance from science can be transferred to business practice. These recommendations are developed through case study research and classified in the four structural areas of Acatech's Industry 4.0 Maturity Index: Resources, Information Systems, Organizational Structure and Culture.
\end{abstract}

\section{Introduction}

A current development in the German energy market is the transition to renewable energies. An analysis by the German Federal Environment Agency shows that energy generation in Germany from renewable energy sources not reached grid parity but also exceeded production from fossil fuels for the first time in 2020 [1]. Onshore wind turbines (WT's) make up the majority share $(41.7 \%)$ of generated energy [2]. Thus, onshore wind turbines are an important factor in securing green, environmentally friendly energy supplies for Germany. However, operators of WT's observe increasing cost pressure [3]. This is chiefly a result of the expiration of the EEG (German renewable energy law) subsidies and age-induced increasing operating and maintenance costs (O\&M costs) [4-6]. The annual O\&M costs of a new turbine are estimated to be at 10 to $15 \%$ of the average total costs per $\mathrm{kWh}$, this share increases to as much as $35 \%$ by the end of its service life [7]. Resulting from this, O\&M costs determine about $25 \%$ of the total costs per $\mathrm{kWh}$ on average [7], resulting in great savings potentials of maintenance optimization measures with the ability to reduce the power generation costs of onshore WT's.

One such promising measure is the implementation of a prescriptive maintenance approach, based on a condition-based maintenance strategy. It is enabled by the advances that accompany the transition to Industry 4.0 [8]. A condition-oriented maintenance strategy performs maintenance activities optimized for time and personnel utilization, influenced by the system and life cycle phase [8]. McKinsey \& Company estimates that this enables a $5-15 \%$ increase in asset availability and a $18-25 \%$ reduction in maintenance costs [9]. However, such optimization relies on detailed knowledge about the WTs condition. This poses challenges for small and medium sized enterprises (SMEs), as they often do not have the capacity for such an evaluation. While previous approaches primarily evaluate operating and structural data from the maintenance history, comments on the condition of the turbine are often neglected. These comments are often contained in non-standardized form in service reports and are documented by service technicians in free text fields.

This is exactly the purpose of the research project "ReStroK". Together with industrial and research partners, the Institute for Industrial Management, FIR is investigating the machine evaluation of both status data 
and maintenance histories, which so far have been used primarily for documentation purposes. The focus is on the avoidance of breakdowns due to progressive wear and tear of individual components by timely maintenance of these components.

So far, the research project has developed an ontology that allows to convert the information contained in free text fields into a usable form [10]. Using the ontology, existing maintenance and service reports are automatically evaluated for issues and potential future breakdowns. This can be utilized to trigger maintenance activities. It also enables the subsequent evaluation of conducted maintenance and service activities by scanning future reports for corresponding indicators for the success or failure of conducted activities. In addition, a function tree for onshore WT's was developed and a measure catalogue with the corresponding maintenance measures was set up. This makes it possible to assign the corresponding cause and countermeasure directly to the error messages. Thus, ReStroK could contribute to the further development of a prescriptive maintenance approach for onshore WT's. The question now arises as to how these scientific findings can be transferred into operational business practice. For a successful implementation, a holistic approach is essential that takes not only technical but also personnel, organizational and social factors into account [11]. Therefore, this paper aims at identifying the key sociotechnical success factors for the successful implementation of a prescriptive maintenance strategy for SME's by original equipment manufacturers (OEM's) and independent service providers (ISP's). These factors are developed through case study research and classified in the four structural areas of Acatech's Industry 4.0 Maturity Index: Resources, Information Systems, Organizational Structure and Culture [12].

\section{Theoretical background}

In this chapter, the technical background of prescriptive maintenance is discussed. In addition, the Industry 4.0 capability clusters on which the success factors are based are explained in more detail.

\subsection{Prescriptive maintenance}

In the context of Industry 4.0, a distinction is made between four different development stages. Stage 1 is about using visibility to understand what is happening and then, in stage 2, using transparency to understand why something is happening. Stage 3 comprises predictive capacity, i.e., the ability to predict what will happen. In the last stage, the knowledge gained from the previous stages is used to achieve adaptability and autonomous self-optimization through data analysis and digital connectivity [12]. According to Davenport, within these stages of development, there are several levels of competitive advantages that can be achieved through business intelligence and analytics (Figure 1). These are divided into the areas of Access \& Reporting and Analytics with sub areas such as Ad hoc reports or Predictive modeling. The greatest competitive advantage can be achieved by using analytics to optimize business activities, such as maintenance [13]. Maintenance activities include upkeep, inspection, repair, and improvement [14]. These activities can be clustered in corrective (after fault occurrence) and preventive maintenance (prior to fault occurrence). Preventive maintenance approaches can be further differentiated. A sub-set are predictive maintenance approaches that aim to carry out maintenance activities derived from data analytics and evaluation of significant system parameters [14]. One extension of such a maintenance approach is prescriptive maintenance.

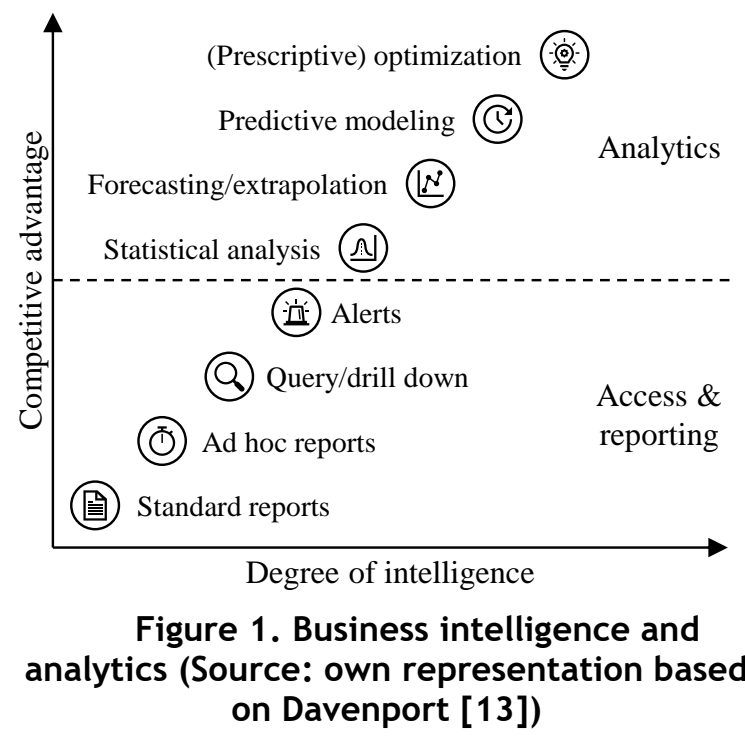

Whereas in a predictive maintenance approach only the condition of the object of maintenance is considered to determine the scope of maintenance activities based on a forecast, a prescriptive maintenance approach considers the object of activities to be a guiding and controlling element for activities as well [15]. Such a maintenance approach would not just include the performance of maintenance activities prior to fault occurrence but also prescribe recommendations for specific actions to prevent breakdowns and reduce downtimes. In order to make such an optimization possible within the framework of prescriptive maintenance, it is necessary to access and collect data of significant parameters as well as to evaluate this data [16]. The insights gained in this way make it possible, 
to prescribe activities to the object of the maintenance strategy.

Onshore WT's collect and transmit a variety of different data that can be used for prescriptive maintenance. These data are the master data of the WT, operating data sourced from Supervisory Control and Data Acquisition Systems (SCADA), which in part comprise hundreds of measuring channels, status and alarm messages as well as event and maintenance data, and data from the component-specific condition monitoring system (CMS) [17]. In previous approaches to maintenance optimization of onshore WT's, mainly SCADA data were evaluated, maintenance data from service reports have hardly been considered so far [1820]. Nevertheless, Faulstich and Hahn already have examined the added value of an evaluation of event data (automatically generated status messages of WT's and maintenance data from service reports) [17]. Therefore, ReStroK has set the goal to increase the amount of available data, which is made possible through the developed ontology [10].

After the collection of data, measures can be prescribed, evaluated for effectiveness on the object of maintenance activities and adapted when needed. This achieves the highest level of maturity and competitive advantages for a system to be self-optimizing [13, 15]. The basic data framework in such a prescriptive maintenance approach based on the four steps data acquisition, data processing, data analysis and decisionmaking is shown in Figure 2.

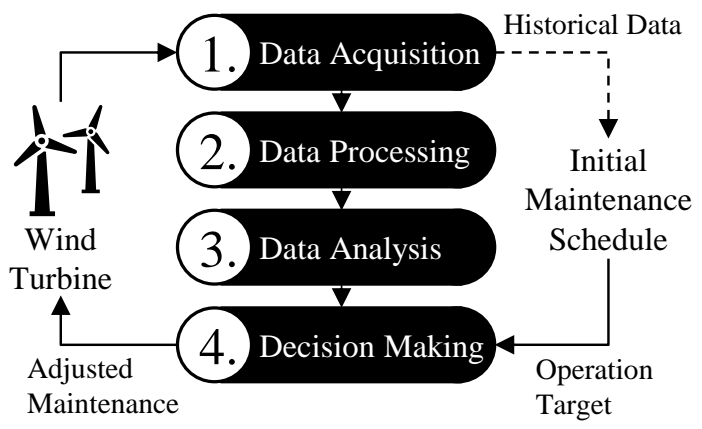

\section{Figure 2. Data framework in a prescriptive maintenance approach (Source: own representation)}

\subsection{Industry 4.0 socio-technical capability clusters}

In order to be able to take the aforementioned development steps of Industry 4.0 and successfully implement such an advanced maintenance approach in practice, a number of capabilities are required. Numerous approaches exist to classify these capabilities
[21-23]; one possible approach was developed by Acatech. According to the approach, the required capabilities can be divided into the following four clusters: Resources, Information Systems, Organizational Structure and Culture. In connection with this, the Acatech Industry 4.0 Maturity Index was also developed to determine the corresponding maturity levels reached by the company under consideration in these clusters [12]. The Acatech approach was chosen because of Acatech's leading role in the development and definition of the concept of Industry 4.0 in Germany.

The first cluster comprises the resources. In the Acatech Industry 4.0 Maturity Index, these are understood to be tangible, physical resources. These include the company's workforce, machinery and equipment, tools, materials, and the final product. The cluster can be further broken down into the two areas of digital capability and structured communication. Digital capability refers to basic requirements for informationbased working and learning. These include the capabilities provide digital competencies, automated data acquisition through sensors and actuators as well as decentralized (pre-) processing of sensor data. The second area covers the structured communication, which refers to the use of communication technology as a tool to create temporary networks enabling resources to interact with each other. This includes the capabilities efficient communication and task-based interface design [12].

The second cluster refers to socio-technical systems in which information is provided based on economic criteria by people, information and communication technology. The main task of an information system is to prepare, process, store and transfer data and information. This cluster can also be broken down into the two areas information processing and integration. Information processing is primarily about the aggregation of data to provide information and material to support decision-making. There are four capabilities that play a role here: automated data analysis, contextualized data delivery, application-specific user interface and resilient IT infrastructure. Integration refers to information systems that are intended to ensure the use of common data throughout the value chain. This requires the following capabilities: horizontal and vertical integration, data governance, standard data interface and IT security [12].

The third cluster deals with the organizational structure. The main focus here is on the implementation of a suitable organizational structure for the transformation into a learning, agile company, the technical requirements of which have already been described in the first two clusters. According to the Acatech Industry 4.0 Maturity Index, this cluster is 
structured in the two areas of organic internal organization and dynamic collaboration within the value network. An organic internal organization is characterized by minimal restrictions and a high degree of individual responsibility. It thus represents an antithesis to the mechanistic organization. The associated capabilities flexible communities, decision rights management, motivational goal systems and agile management reflect this. The second area dynamic collaboration within the value network aims at an automated and seamless exchange of information between different companies. This enables the companies to form dynamic cooperations and provides increased transparency regarding the market. This area includes the capabilities focus on customer benefits as well as cooperation within the network [12].

The fourth and final cluster comprises Culture and accordingly deals with the behavior of a company's employees. To successfully achieve the transformation to an agile company, which makes use of Industry 4.0, it is necessary to change the mentality of all employees. In this context, a distinction between the areas of willingness to change and social collaboration is made. Willingness to change includes five capabilities that must be acquired by employees of learning, agile companies. These include the ability to recognize the value of mistakes, openness to innovation, databased learning and decision-making, continuous professional development and shaping change. Only when all these skills are present will they result in willingness to change. Social collaboration, on the other hand, is about accelerating knowledge sharing within the organization. This is enabled by the combination of the three capabilities democratic leadership style, open communication and confidence in processes and information systems [12].

\section{Research Method}

Maintenance of onshore WT's is characterized by complex relationships between OEMs, operators, and independent service providers (ISPs). This is primarily due to conflicting interests (OEMs and ISPs are in direct competition and want to sell their maintenance contracts) [24]. This results in little to no exchange of data and/or knowledge, which would be essential for targeted optimization of maintenance. In order to understand the individual challenges faced by the relevant groups, they must be approached individually.

Our chosen research method consists of an exploratory multiple case study. The research design follows the phenomenological approach of qualitative research [25]. The study relies on a non-random purposive sampling based on market share (OEMs) and selection based on size (operators \& ISPs) as well as focus on project members of ReStroK. In the selection of the companies considered, the focus was also on the great diversity of the companies to be able to cover the conflicts of interest already described. In total, five OEMs, operators, and ISPs were surveyed, with 8 interviewees, mostly from middle to higher management (Table 1). All interviewed individuals play a key role in the technical management and development of digitalization in their respective company.

The interviews were conducted in a semi-structured manner with a duration of 60 minutes per interview. Due to pandemic restrictions, all interviews were performed online. The interviews were utilized for the iterative identification and validation of success factors for the socio-technical implementation of a prescriptive maintenance approach. For this purpose, a guideline for conducting the interviews was developed based on the Acatech framework. The guideline consisted of three sections. First, questions were asked about the potential procedure for implementing such a maintenance approach at the target company. Then the focus was shifted on the capabilities required for this. Finally, the relevance of the factors developed in the Acatech framework was evaluated in the four clusters. The evaluation was carried out qualitatively based on five evaluation levels: 0 - not relevant, 1 - of little relevance, 2 - somewhat relevant, 3 - very relevant, 4 - of critical relevance. The results were captured in field notes, supported by audio-recordings if the interviewee agreed.

We chose this research method in order to obtain comprehensive expert knowledge from individual subjects and to be able to determine individual perspectives. The differentiated description of content and/or processes makes it possible to do justice to the competitive situation between OEMs and ISPs and to consider this appropriately. All of these criteria can best be implemented by means of interview-based, qualitative research [26]. 
Table 1. Overview and specifics of case study companies and conducted interviews

\begin{tabular}{|c|c|c|c|}
\hline Case ID & Role & Size [\# employees] & Interviewees' Position \\
\hline OEM1 & OEM & $1.000-5.000$ & Senior Management, Management, Employee \\
\hline OEM2 & OEM & $>10.000$ & Management \\
\hline OP1 & Operator & $1-10$ & Senior Management \\
\hline OP2 & Operator & $1-10$ & Director \\
\hline ISP1 & ISP & $100-500$ & Management, Senior Management \\
\hline
\end{tabular}

\section{Case studies}

The case studies serve the iterative development and implicit validation of the identified success factors for the implementation of a prescriptive maintenance approach. They allow for an in-depth analysis of a realworld phenomenon under the consideration of environmental influences, which (other than in an experiment) cannot be delimited or controlled.[27]

Three case studies were selected to be discussed in detail in the following (OEM1, OP1, ISP1). The selections of cases for detailed discussion in this paper were based on company types (one study for OEMs, one for operators, and one for independent service providers) as well as geographical proximity. The case studies are presented, describing the maintenance implementation approaches adopted by each company and addressing the different factors presented in the framework.

\subsection{Case 1 (OEM1)}

The presented OEM use case (OEM1) consists of an international public company headquartered in Western Europe. It operates six manufacturing plants across the globe with an output capacity of $\sim 16,000 \mathrm{MW}$ per year. It produces WT components and provides maintenance services for its customers.

The OEM aims to build on advantages it already possesses in regards to customer relations, product knowledge and access to resources (e.g., spare parts, skilled employees) when delivering a maintenance approach to a client. Another factor for success is a rich internal database to develop solutions for the "fleet". This focus on internal strengths has led to major investments in digital competencies of its personnel, even setting up entire departments dedicated to data analytics and rigorously ensure continuous learning of its workforce. In addition to that, it was stressed how important a change management mindset was important for success. By focusing on strong capabilities by its workforce but also the ability to automatically analyze large amounts of data, it has developed a wide offering of services, such as predictive analytics or the ability to perform root cause analyses for clients. They also aim to make the data as conceivable as possible by putting focus on its presentation in task based interface designs, however application specific interfaces were valued a bit less, as they only "improve the quality of life" and were not deemed critical for success. These services are accompanied by the need to perform diligent data governance as "it doesn't matter how nice a report looks; it also needs to be correct". Another key factor, which was discussed in detail, was the aspect of IT security, as legal risks could be quite severe, as well as a resilient IT infrastructure, which was characterized as "a must" by one interviewee. The company continuously seeks to expand its maintenance solution portfolio and aims to further its abilities to offer prescriptive maintenance capabilities as an expansion to its current portfolio.

\subsection{Case 2 (OP1)}

The presented operator use case (OP1) consists of a citizen-financed wind park in Western Germany. It was founded in the early 2000 s, operates 5 WT's (1MW each, all from the same OEM) and produces $\sim 8 \mathrm{GWh} /$ year. The company is considered an SME.

For the first 15 years, the company utilized a maintenance package (contract duration: 15 years) offered by the OEM. This initial choice regarding provider and scope was primarily made due to the unavailability of alternative providers as well as a distinctive lack of knowledge regarding the maintenance needs for the owned WT's. As stated by the interviewee "you could only chose the OEM, simply because [the OEM] didn't share any information such as 
asset drawings, maintenance plans or spare parts with ISP's". After the termination of the initial maintenance and service contract, the company evaluated the asset history to determine the requirements for any future maintenance and service contract. In addition to this evaluation, the company collected several key factors to facilitate the choice for the next provider. While no explicit framework was used, the factors served as a foundation for the decision on a provider and maintenance and service strategy.

The overarching goals for such a strategy were to ensure WT availability, performance, and low maintenance costs. The technological advances and opportunities provided by industry 4.0 (such as databased preventive maintenance) should also be utilized to achieve these goals. Thus, the considerations included other factors such as the capability to utilize data to carry out improved maintenance activities. Other factors (such as communication) were not considered in depth. Eventually, the OEM was chosen again, however in a more limited role, with a less extensive maintenance agreement, however with additional digital services such as remote monitoring of the WT's and the automatic triggering of maintenance activities. During the implementation of further digital capabilities it was observed that (corporate-) cultural and organizational factors were not of great importance for the success of the implementation. However, the digital capabilities of the OEM as well as the integration of available data into systems along the value chain proved to be decisive for success. Overall, the adjustment has been considered successful based on lowered costs, improved WT availability and performance.

Currently the company is evaluating opportunities to further improve maintenance activities by establishing a prescriptive maintenance system. As this is mostly considered an extension of already received services, the success factors are considered similar.

\subsection{Case 3 (ISP1)}

The presented service provider use case (ISP1) consists of an SME from Central Germany. It was founded in the early 2000s and serves as operations manager as well as maintenance service provide for its clients. It operates $\sim 360$ WT's and maintains $\sim 300$ WT's independently from OEM's across Germany.

When implementing maintenance solutions, the overarching goal is the optimal return on invest (RoI) of the asset to the customer, the WT operator. Especially for the implementation of data based maintenance services, the company has identified certain factors as critical. As an example, it has worked on developing proprietary tools to enable the automated data collection and initial evaluation from a range of WT OEM's. It has also focused on building up digital capabilities within the company to be able to handle data-analytics, as this has supplemented the need for WT-specific hardware knowledge to carry out maintenance operations. The tools were set up with a task based interface as "critical data can easily be overlooked in cluttered systems", according to the interviewees. The company's information systems were expanded significantly over the past 10 years to keep pace with the technical evolution of WT's and the changes in the data they provide. The focus on data governance has also increased as the amounts of collected data have grown significantly, leading to an even greater dependence on automated data quality control. The organizational structure has been adapted by including employees with data science capabilities into the capability clusters, setup for each manufacturer of serviced WT's to reflect the increased focus on data-based maintenance activities. The company aims to foster a culture of open communication and willingness to embrace innovations, as the ISP needs to stay up to date with the technical developments of a wide range of OEM's. A future project is the development of a prescriptive maintenance offering for clients using data generated by the WT as well as maintenance technicians.

\section{Discussion}

The results show that factors recognized and addressed by companies can be matched to the sociotechnical capability clusters defined in the Acatech framework, which was discussed in chapter 2. The highlights of the results are displayed in Figure 3. 


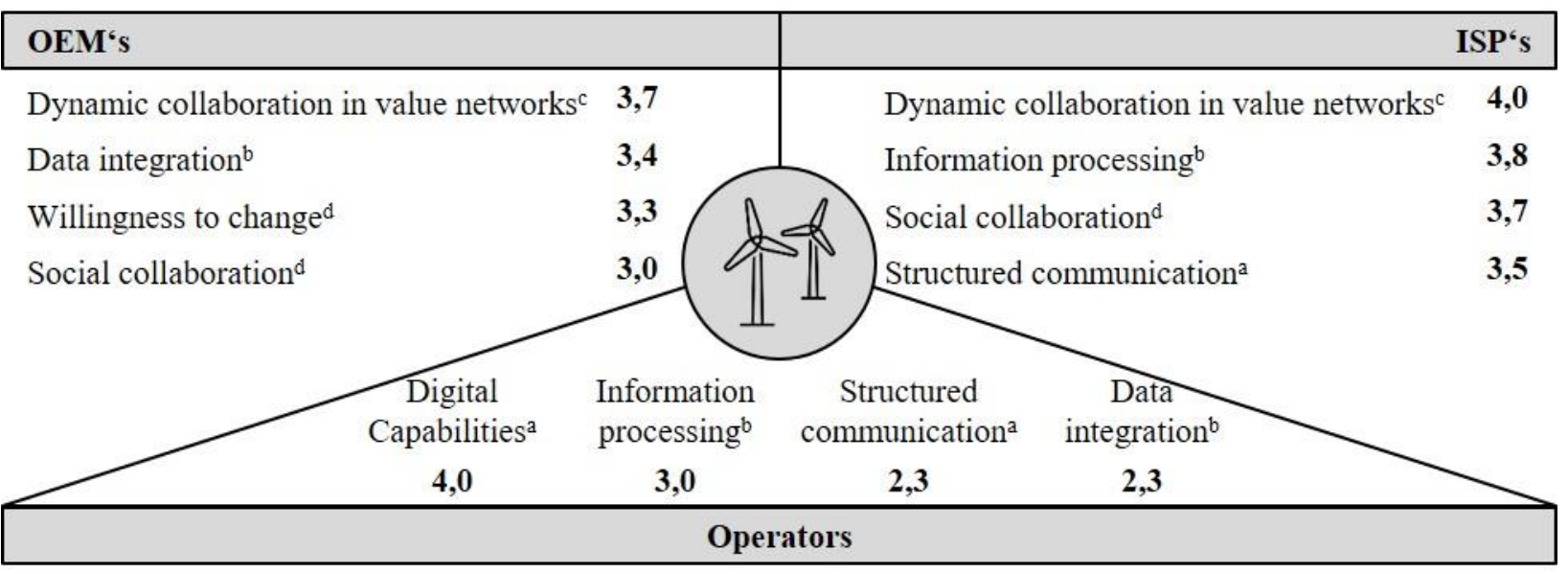

(a) Ressources; (b) Information Systems; (c) Organizational Structure; (d) Culture

0 : Not relevant; 1: Of little relevance; 2: Somewhat relevant; 3: Very relevant; 4: Of critical relevance

Figure 3. Identified key success factors and corresponding ratings

The result shows, that the actors have identified and prioritized varying factors for the successful implementation of a prescriptive maintenance approach. The largest differences lie in the prioritization of organizational factors, especially those concerning the collaboration in value networks by OEM's and ISP's, whereas operators value digital capabilities and structured communication more highly. In the following, each cluster will be discussed in detail.

Resources: The build-up of digital capabilities is of great importance for all actors. Digital competencies of employees are considered critical by operators, OEM's and ISP's as this is a prerequisite to handle the data related tasks such as data analysis and interpretation that prescriptive maintenance requires. The ability for automated data acquisition through sensors and actuators is the factor that is valued highest by all actors, as automation enables the capture of the amounts of data required for prescriptive maintenance. The preprocessing of the collected data rated slightly higher by the operators than by OEM's and ISP's, with it being critical for the former. Actors that execute maintenance, OEM's and ISP's, generally rated structured communication higher. While factors, such as efficient communication were expressed to be "nice to have" factors by operators in interviews, especially ISP's, place great value in the ability to communicate efficiently with their clients to achieve strong results and maintain relationships. The set-up of task based interface designs is mostly considered somewhat relevant, mostly considered an enabler for available tools. ISP's prioritize this cluster the most, mostly due to the strong focus on communications, followed closely by operators.

Information systems: The cluster is made up of the sub-clusters information processing and integration. Just like the automated data acquisition, the automated data analysis is assessed as critical for the successful implementation. It should be noted, that OEM's were most confident in their current capabilities in this regard, compared to ISP's and operators (which oftentimes expressed the lack of resources to be a challenge in this regard in interviews). Operators rated the contextualized data delivery the highest, as very relevant, stating in interviews that this offered the greatest possibility for them to gain an improved understanding over their assets. The resilience of the employed infrastructure was rated as very relevant or critical across the board, as all actors were aligned in placing importance on the ability to handle disruptions. The sub-cluster integration showed greater differences. OEM's and ISP's consistently rated the sub-cluster to be more important than operators. With factors such as IT security, some operators did not express worries on possible data breaches, while ISP's and OEM's cited both the need to protect WT's from manipulation but also liability issues as reasons for great focus on this topic. The governance of data and the resulting issues e.g. of data quality were also considered to be more of a "doer's" problem by operators. This was evident with the average score assigned by operators was a 2,5 while ISP's and OEM's rated this factor with a 4 . The ratings for standard data interfaces were quite similar with similar underlying reasons. The horizontal and vertical integration of systems with the resulting single source of truth is 
considered important by all actors. Again, with greater focus by ISP's and OEM's with one operator stating in an interview that "he will worry about it once he achieves this for his own systems".

Organizational structure: The placed importance on organizational structure varied greatly between actors. Flexible communities were valued strongly by ISP's, as they face the challenges of servicing a multitude of differing WT's from a range of OEM's. Operators did not consider this factor to be relevant at all, while OEM's also did not consider it to be very important. This was almost reversed for decision rights. As the investigated operators were all SME's and thus without extensive hierarchical structures, they did not consider the decentral management of decision rights to be as important as ISP's (3) or OEM's (1,5). A motivational goal system was considered to be important for a strong corporate culture by all actors, but less important for the implementation of a maintenance strategy, thus scoring quite low across the board. The feedback regarding agile management approaches was also mixed, while OEM's regarded it quite highly and important to enable teams to carry out work efficiently, it came with a caveat that the use and its effectiveness during an implementation depended on the client and his preferences. The collaboration in value networks was rated very high by OEM's and ISP's as they viewed the collaboration critical for success, while the surveyed operators did not regard value networks as highly.

Culture: The cultural cluster and its factors was rated highest by OEM's and second-lowest by operators. A democratic leadership style was considered to be critical by ISP's and very important by OEM's, while OEM's did not consider it to be important for the implementation of a maintenance system for them. Open communication was perceived to be mostly "relevant when problems occur" by operators but critical for success by both, OEM's and ISP's. Operators rated confidence in processes lower than the other actors, which deemed this factor very important. Overall, it can be observed, that clusters often considered "soft" in public discourse, such as culture or organizational structure scored higher in larger, more mature companies (with OEM's valuing it slightly higher than ISP's).

Limitations: The research carried out is associated with some limitations. For example, the total number of people interviewed is relatively small due to time restrictions. In addition, several people from the same company were interviewed in some cases. However, the independent questioning ensured that different perspectives could be included and that there was no unintentional bias. Furthermore, the focus in the selection of the interview partners was primarily on Europe and on the implementation of such an approach in the European markets. This focus was mostly due to the availability of interview partners and further research should be conducted to investigate geographical differences. Possible differences in success factors could be found in varying education levels or varying degrees of government regulation, for example. Also when interpreting the results, the differing backgrounds (e.g. education or job profiles) of the interviewee's need to be considered. Overall, it is also not yet possible to perform a concrete mapping of the identified success factors to the potential technical and economic benefits. Thus, no final theoretical concept was developed, but a first step towards the practical implementation of such maintenance approaches was made and additional research needs were identified.

Table 2. Overview over the results by actors in clusters and sub-clusters

\begin{tabular}{|c|c|c|c|c|}
\hline Cluster & Sub cluster & Operators & OEM's & ISP's \\
\hline Resources & Digital Capability & 4,0 & 2,6 & 2,7 \\
\hline Resources & $\begin{array}{c}\text { Structured } \\
\text { Communication }\end{array}$ & 2,3 & 3,2 & 2,8 \\
\hline Information systems & Information processing & 3,0 & 2,8 & 2,6 \\
\hline Information systems & Integration & 2,3 & 3,4 & 3,4 \\
\hline Organizational structure & $\begin{array}{c}\text { Organic internal } \\
\text { organization }\end{array}$ & 0,5 & 2,2 & 2,0 \\
\hline Organizational structure & $\begin{array}{c}\text { Dynamic collaboration } \\
\text { in value networks }\end{array}$ & 1,0 & 3,7 & 3,5 \\
\hline
\end{tabular}




\begin{tabular}{|l|c|c|c|c|}
\hline Culture & Social collaboration & 0,7 & 3,2 & 3,3 \\
\hline Culture & Willingness to change & 1,2 & 3,3 & 3,2 \\
\hline
\end{tabular}

\section{Conclusion and Outlook}

The aim of this paper was to investigate and validate sociotechnical success factors for the implementation of a prescriptive maintenance approach for onshore WT operators. This effort has been undertaken to provide especially SME's with some guidance in implementing such an approach. Accordingly, the paper did not develop a final theoretical concept, but rather a practical guideline as well as identified additional need for further research.

First, it has been shown that certain factors, such as strong digital capabilities, are of critical importance for all stakeholders (operators, OEM's and ISP's) to ensure a successful implementation. Some other factors, such as cultural or organizational factors, have been shown to be of varying importance, with the executing parties (ISP's and OEM's) putting higher priorities on such factors. By validating these factors in the case studies and corresponding interviews, this paper provides a solid foundation for operators, OEM's and ISP's to develop further detailed approaches based on them in the future.

It must be noted, that while this paper yielded first insights and substantiated findings, a higher number of

\section{References}

[1] Umweltbundesamt (Ed.), "Erneuerbare Energien in Zahlen", March 4, 2021. Retrieved from https://www.umweltbundesamt.de/themen/klimaenergie/erneuerbare-energien/erneuerbare-energien-inzahlen\#uberblick. Accessed June 11, 2021.

[2] Bundesministerium für Wirtschaft und Energie (BMWi) (Ed.), "Erneuerbare Energien in Zahlen: Nationale und internationale Entwicklung im Jahr 2019", October, 2020. Retrieved from:

https://www.bmwi.de/Redaktion/DE/Publikationen/En ergie/erneuerbare-energien-in-zahlen2019.pdf?_blob=publicationFile \&v=6. Accessed June 11, 2021.

[3] F. Urbansky, "Die auslaufende EEG-Förderung wirft ihre Schatten voraus", December 21, 2018. Retrieved from https://www.springerprofessional.de/erneuerbareenergien/erneuerbare-energien-gesetz/die-auslaufendeeeg-foerderung-wirft-ihre-schatten-voraus/16345856. Accessed June 6, 2021. cases from a greater range of company sizes and geographies would be beneficial to enhance the understanding of success factors as well as their interdependencies and the robustness of the research results. In addition, further research still needs to be conducted to detail the relationships, goal-sets and dependencies between the players in the industry. Another aspect to be explored in the future is the mapping of the identified most relevant factors to potential technical and economic benefits.

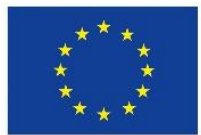

EUROPEAN UNION Investing in our Future European Regional Development Fund $20 \frac{14}{2}$ EFRE.NRW Investitionen in Wachstum und Beschäftigung

\section{Acknowledgements}

This project was funded by the European Regional Development Fund (EFRE) under the project number EU-2-2-029.

[4] I. Staffell, and R. Green, "How does wind farm performance decline with age?", Renewable Energy, 66, 2014, pp. 775-786.

[5] J. Dai, W. Yang, J. Cao, D. Liu, and X. Long, "Ageing assessment of a wind turbine over time by interpreting wind farm SCADA data", Renewable Energy, 116, 2018, pp. 199-208.

[6] L. Ziegler, E. Gonzalez, T. Rubert, U. Smolka, and J.J. Melero, "Lifetime extension of onshore wind turbines: A review covering Germany, Spain, Denmark, and the UK", Renewable and Sustainable Energy Reviews, 82, 2018, pp. 1261-1271.

[7] Morthorst, P.E., H. Auer, A. Garrad, and I. Blanco, Wind Energy the Facts: The Economics of Wind Power. Retrieved from https://www.wind-energy-thefacts.org/images/chapter3.pdf. Accessed 2 April 2021.

[8] IZP, IWES, SAG Erwin Peters GmbH, and ENERTRAG AG (Eds.), Erhöhung der Verfügbarkeit von Windkraftanlagen EVW-Phase I. Teilprojekt: Entwicklung von zuverlässigkeitsbezogenen Betriebsund Instandhaltungsstrategien für die Windenergie 
unter besonderer Berücksichtigung der OffshoreBedingungen. EVW I, Förderkennzeichen 0327574, 2009, 132 pp. Retrieved from http://www.windevw.de/fileadmin/Dokumente/Abschlussberichte/EV W_I_Abschlussbericht/EVW_Phase_I_Abschlussberic ht.pdf. Accessed 11 June 2021.

[9] Bradburry, S., B. Carpizo, D. Horah, and J. Thibert, "Digitally enabled reliability: Beyond predictive maintenance", October, 2018. Retrieved from: https://www.mckinsey.com/ /media/mckinsey/busines s\%20functions/operations/our\%20insights/digitally\%2 0enabled $\% 20$ reliability $\% 20$ beyond $\% 20$ predictive $\% 20$ maintenance/digitally-enabled-reliability-beyondpredictive-maintenance.pdf?shouldIndex $=$ false. Accessed July 29, 2021.

[10] Strack, B., M. Lenart, J. Frank, and N. Kramer, "Ontology for maintenance of onshore wind turbines", Forschung im Ingenieurwesen (Forschung im Ingenieurwesen), 66(B), 2021, pp. 775-782.

[11] Abel, J., H. Hirsch-Kreinsen, S. Steglich, and T. Wienzek, Akzeptanz von Industrie 4.0, acatech, Munich, 2019. Retrieved from https://www.plattformi40.de/PI40/Redaktion/DE/Downloads/Publikation/ak zeptanz-industrie40.pdf?_blob=publicationFile \&v $=6$. Accessed September 3, 2021.

[12] Schuh, G., R. Anderl, R. Dumitrescu, A. Krüger, and M. ten Hompel, Industrie 4.0 Maturity Index: Managing the Digital Transformation of Companies UPDATE 2020, acatech, Munich, 2020. Retrieved from https://en.acatech.de/publication/industrie-4-0maturity-index-update-2020/download-pdf?lang=en. Accessed June 11, 2021.

[13] Davenport, T.H., and J.G. Harris, Competing on analytics: The new science of winning, Harvard Business School Press, Boston (MA), 2007.

[14] DIN Deutsches Institut für Normung e. V. (Ed.), Grundlagen der Instandhaltung, Beuth, Berlin, 01.040.03; 03.080.10(31051:2019-06), June, 2019.

[15] Franzen, J., J. Stecken, R. Pfaff, and B. Kuhlenkötter, "Using the Digital Shadow for a Prescriptive Optimization of Maintenance and Operation", in Clausen, U, Langkau, S, and Kreuz, F, Eds. Advances in Production, Logistics and Traffic, Springer, Cham, 2019, pp. 265-276.

[16] Clausen, U., S. Langkau, and F. Kreuz, eds., Advances in Production, Logistics and Traffic, Springer International Publishing, Cham, 2019.

[17] Faulstich, S., and B. Hahn, "Erkenntnisse aus digitalisierten Daten von über 3000 Windenergieanlagen im Betrieb", in VDI Wissensforum GmbH, Ed. VDI-Fachtagung 'Schwingungen von Windenergieanlagen 2019', Bremen, 6/4/2019-6/5/2019, VDI Verlag, Düsseldorf, 2019. Retrieved from https://www.researchgate.net/publication/334051041. Accessed 11 June 2021.

[18] Zhou, A., D. Yu, and W. Zhang, "A research on intelligent fault diagnosis of wind turbines based on ontology and FMECA", Advanced Engineering Informatics, 29(1), 2014, pp. 115-125.

[19] Papadopoulos, P., and L. Cipcigan, "Wind turbines' condition monitoring: an ontology model", in 2009
International Conference on Sustainable Power Generation and Supply: SUPERGEN 2009, IEEE, Ed, Nanjing, 4/6/2009 - 4/7/2009, pp. 1-4. doi: 10.1109/SUPERGEN.2009.5430854

[20] Ertek, G., X. Chi, A.N. Zhang, and S. Asian, "Text mining analysis of wind turbine accidents: An ontology-based framework", in 2017 IEEE International Conference on Big Data (Big Data), I. Staff, Ed, Boston (MA), 12/11/2017 - 12/14/2017, pp. 3233-3241, doi: 10.1109/BigData.2017.8258305.

[21] Winkelhaus, S., A. Sutter, E. Grosse, and S. Morana, "Soziotechnische Systeme: Der Mensch in der Industrie 4.0", Industrie 4.0 Management, 37(3), 2021, pp. 45-48.

[22] Block, C., S. Freith, N. Kreggenfeld, F. Morlock, C. Prinz, D. Kreimeier, and B. Kuhlenkötter, "Industrie 4.0 als soziotechnisches Spannungsfeld", Zeitschrift für wirtschaftlichen Fabrikbetrieb, 110(10), 2015, pp. 657-660.

[23] Stocker, A., M. Rosenberger, M. Resztej, and S.A. Damalas, "Erfolgsfaktoren bei der Einführung Digitaler Technologien im Kontext von Industrie 4.0", September, 2020. Retrieved from: https://www.researchgate.net/publication/344430217. Accessed September 3, 2021.

[24] Parbs, H., Fragmentierung eines Service Ecosystem: Eine Grounded Theorystudie zur Instandhaltung in der Windenergiebranche, Bremen, University, Dissertation, 2017. Retrieved from https://media.suub.unibremen.de/bitstream/elib/1408/1/00106483-1.pdf. Accessed 11 June 2021.

[25] Creswell, J.W., Qualitative inquiry \& research design: Choosing among five approaches, Sage, Los Angeles (CA), 2018.

[26] Misoch, S., Qualitative Interviews, de Gruyter Oldenbourg, Berlin, 2015.

[27] H.-G. Ridder, "The theory contribution of case study research designs", Business Research, 10(2), 2017, pp. 281-305. 